\title{
Aire Acondicionado Solar, para Conjunto de Viviendas en Mexicali, México
}

\author{
Aníbal Luna ${ }^{1}$, Nicolás Velázquez ${ }^{2}$, Ricardo Gallegos ${ }^{1}$ y Gonzalo Bojorquez ${ }^{1}$ \\ Universidad Autónoma de Baja California, (1) Facultad de Arquitectura y Diseño, (2) Instituto de \\ Ingeniería, Calle de La Normal e Ignacio López Rayón s/n, Unidad Mexicali, 21280 Mexicali, \\ Baja California-México (e-mail: \{anibal, nicolasvelazquez, rikrdo, gonzalobojorquez\}@uabc.mx)
}

\begin{abstract}
Resumen
El objetivo del presente trabajo es dimensionar y evaluar un sistema de enfriamiento por absorción $\mathrm{LiBr}-\mathrm{H}_{2} \mathrm{O}$ asistido con energía solar y gas natural, para climatizar un conjunto de viviendas en Mexicali, Baja California, México. El análisis de comportamiento horario, diario y mensual en el periodo con mayor demanda de enfriamiento se realiza con el programa TRNSYS 16 y se busca la menor área de captación y la mayor fracción solar. Esto se traduce en el mínimo costo de operación e inversión inicial. Se encontró que el sistema genera condiciones del espacio entre confortablemente caliente y confortablemente frío, según la norma ISO 7730 (2005). Esto se logra a partir de $287 \mathrm{~m}^{2}$ de tubos evacuados, que aportan el 90\% de la energía requerida. El sistema es técnicamente factible, ya que cubre la demanda de enfriamiento durante el periodo de verano y requiere un área de colección similar a la disponible en el conjunto de viviendas.
\end{abstract}

Palabras clave: absorción de $\mathrm{LiBr}-\mathrm{H}_{2} \mathrm{O}$, fracción solar, confort térmico, energía solar, climatización

\section{Solar Air Conditioning for a Housing Compound in Mexicali, Mexico}

\begin{abstract}
The objective of the present work is to dimension and evaluate an absorption $\mathrm{LiBr}-\mathrm{H}_{2} \mathrm{O}$ cooling system assisted by solar energy and natural gas for air conditioning of a group of housings in Mexicali, Baja California, Mexico. The analysis of the hourly, daily and monthly operating performance in the period with more cooling demand is carried out by mean of TRNSYS 16, and it is seeking the least collection area and the highest solar fraction; that which is translated in the minimum operation cost and initial investment. It was found that the system generates conditions of the space between comfortably warm and comfortably cold, according to ISO 7730 (2005), with $287 \mathrm{~m}^{2}$ of evacuated tubes which contribute $90 \%$ of the required energy. The system is technically feasible, since it covers the cooling demand during the summer period and it requires a similar collecting area to the available one in the housing compounds.
\end{abstract}

Keywords: absorption $\mathrm{LiBr}-\mathrm{H}_{2} \mathrm{O}$, solar fraction, thermal comfort, solar energy, air conditioning 


\section{INTRODUCCIÓN}

El clima cálido seco de Mexicali, Baja California, México, provoca grandes demandas de energía eléctrica para mantener los espacios en confort térmico. Las temperaturas promedio máximas llegan a $42^{\circ} \mathrm{C}$ (con máximas extremas de $49^{\circ} \mathrm{C}$ ). La alta disponibilidad de energía solar, representa una oportunidad para el uso de sistemas térmicos de enfriamiento (Hans-Martin, 2007; Papadopoulos et al., 2003), y contribuye en la disminución del efecto invernadero por emisiones de $\mathrm{CO}_{2}$. Además de reducir daños a la capa de ozono por clorofluorocarbonos (CFCs) (Florides et al., 2002a).

Los sistemas de mayor predominancia, son los de bromuro de litio-agua $\left(\mathrm{LiBr}-\mathrm{H}_{2} \mathrm{O}\right)$ y los de amoniaco-agua $\left(\mathrm{NH}_{3}-\mathrm{H}_{2} \mathrm{O}\right)$ (Srikhirin et al.,2001). En la última década se han hecho estudios de simulación de estos sistemas, (Assilzade et al., 2005; Florides et al., 2002a y b), en los que se establecen estrategias y métodos de optimización para selección de: dimensionamiento de tanque, tipo de colector, área de colección, inclinación de colector y temperatura óptima de operación del calentador auxiliar. Otro estudio experimental es el de Syed et al. (2005), los cuales estudian un sistema de enfriamiento solar (SES) con capacidad de $35 \mathrm{~kW}$, operado en las horas con disponibilidad de recurso solar. Sin embargo, para establecer las mejores condiciones de operación del sistema, es necesario conocer el comportamiento simultáneo de todos los subsistemas de la unidad de aire acondicionado solar, a lo largo de todo el periodo de demanda de enfriamiento. El objetivo del presente trabajo es realizar el dimensionamiento y análisis detallado de factibilidad técnica operativa de un sistema de enfriamiento termosolar por absorción de $\mathrm{LiBr}-\mathrm{H}_{2} \mathrm{O}$. El cual será utilizado en un conjunto habitacional de construcción en serie, cuyo prototipo representa el $85 \%$ de un fraccionamiento compuesto por 9,500 viviendas, construidas entre 1995 y 2004 en Mexicali, Baja California, México.

El comportamiento térmico de la envolvente y la influencia que propicia el sistema de enfriamiento solar en las condiciones de confort térmico, se evalúa a través del programa TRNSYS 16; operado a partir de un archivo de año típico meteorológico (TMY). Para el análisis del comportamiento operativo de los subsistemas, se realiza un estudio horario, diario y mensual del periodo de demanda de enfriamiento. A partir de dicha información se establece el potencial de aplicación de la tecnología de climatización solar de un conjunto habitacional de 5 viviendas.

\section{SISTEMA DE ENFRIAMIENTO TERMOSOLAR}

En la figura 1, se muestra el esquema del sistema de enfriamiento solar, compuesto por 7 subsistemas: 1) Edificación, 2) Captación solar y almacenamiento térmico, 3) Suministro de agua caliente, 4) Agua de enfriamiento, 5) Sistema de enfriamiento, 6) Agua fría y 7) Aire frío.

La unidad utilizada en el estudio, es un equipo YASAKI de $35.2 \mathrm{~kW}$ (126720 kJ/h), modelo WFCSC10 que emplea la solución de $\mathrm{LiBr}-\mathrm{H}_{2} \mathrm{O}$ como fluido de trabajo. Dicha unidad opera a través de un fluido caliente entre 75 y $101{ }^{\circ} \mathrm{C}$ (agua-etilenglicol). El fluido caliente produce la ebullición de la solución de $\mathrm{LiBr}-\mathrm{H}_{2} \mathrm{O}$ y posteriormente conduce el vapor de agua generado (refrigerante) hacia el condensador. En el condensador, el vapor es enfriado y condensado por el agua de la torre con temperatura de entre 24 y $31^{\circ} \mathrm{C}$. La torre extrae el calor tanto del condensador como del absorbedor. El refrigerante condensado, es dirigido en forma líquida al evaporador a través de una válvula de expansión que mantiene la baja presión en la sección del evaporador y absorbedor, lo que provoca que el agua evapore a baja temperatura.

Por otro lado, la corriente de agua a enfriar entra al evaporador a temperatura entre 7 y $13^{\circ} \mathrm{C}$, lo que representa suficiente gradiente de temperatura para evaporar el refrigerante procedente del condensador; a la vez que se provoca el enfriamiento de la corriente de agua que pasa por el evaporador. De forma simultánea, la solución en el absorbedor procedente del generador atrapa el vapor de agua y se obtiene una solución diluida de $\mathrm{LiBr}-\mathrm{H}_{2} \mathrm{O}$ que es llevada por medio de una bomba al generador. Cuando la solución de $\mathrm{LiBr}-\mathrm{H}_{2} \mathrm{O}$ es llevada por la bomba hacia el generador, pasa a través de un intercambiador de calor que tiene la función de enfriar la solución procedente del generador y calentar la solución que va hacia el generador, con lo que se obtiene integración energética interna. Finalmente, la solución diluida llega al generador para iniciar de nuevo el ciclo. 


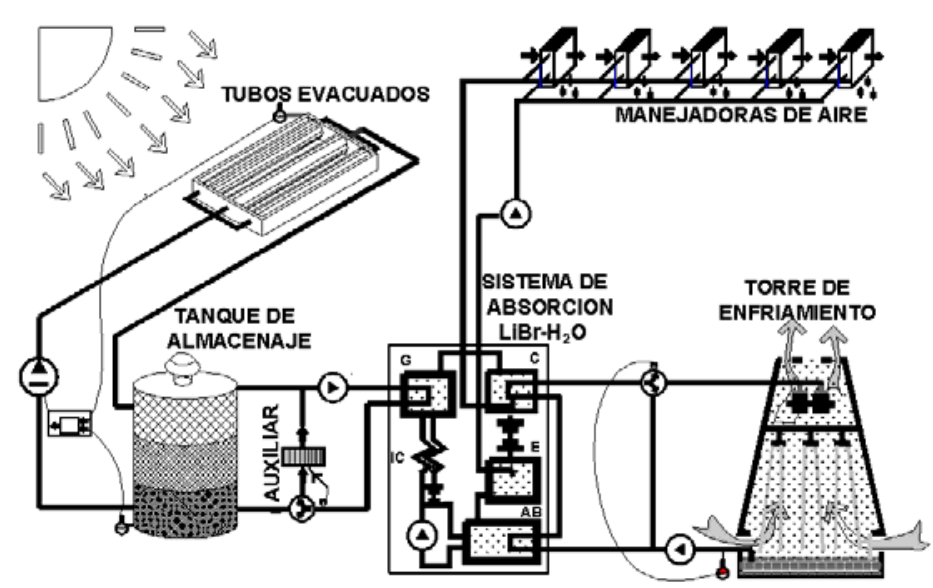

Fig. 1: Sistema de enfriamiento solar

\section{MÉTODO DE SIMULACIÓN}

Para dimensionar y conocer el comportamiento operativo del sistema de enfriamiento solar, se elaboró una estrategia de simulación utilizando TRNSYS 16. El simulador, en general cuenta con una estructura modular que divide el sistema en una serie de "types" (componentes), que son interconectados entre si y compilados a través la interface TRNSYS Studio. En la tabla 1, se muestran los componentes utilizados en la simulación.

Tabla 1: Componentes utilizados para simulación de sistema de absorción

\begin{tabular}{|l|c|}
\hline \multicolumn{1}{|c|}{ COMPONENTE } & No. Componente \\
\hline Archivo meteorológico & 109 \\
\hline Colector de tubos evacuados & 538 \\
\hline Tanque almacenamiento térmico & 4 \\
\hline Bomba de flujo variable & 110 \\
\hline Control variable & 22 \\
\hline Control de encendido / apagado & 2 \\
\hline Divisor y mezclador de flujo & 11 \\
\hline Torre de enfriamiento & 51 \\
\hline Sistema de absorción & 107 \\
\hline Bomba de flujo constante & 3 \\
\hline Calentador auxiliar & 6 \\
\hline Manejadora de aire & 508 \\
\hline Sombras y partesoles & 34 \\
\hline Propiedades del aire ambiente & 33 \\
\hline Temperatura efectiva del cielo & 69 \\
\hline Edificio & 56 \\
\hline
\end{tabular}

Con la finalidad de simular el sistema de aire acondicionado solar bajo condiciones similares a la operación real, la alimentación de la información de los equipos se realiza con datos técnicos proporcionados por los fabricantes de los mismos. Una vez seleccionados los equipos que componen el sistema de enfriamiento solar, se procede al análisis detallado, y se sigue el método mostrado en la figura 2, con la finalidad de verificar si el sistema cumple con las condicionantes siguientes:

1) Temperatura de salida del agua caliente del tanque entre 75 y $101{ }^{\circ} \mathrm{C}$, si esta condición se cumple la fracción solar debe ser mayor al $90 \%$, si las condicionantes anteriores no se cumplen, la energía faltante se suministra a través de un sistema de calentamiento auxiliar.

2) Temperatura de alimentación del agua de enfriamiento entre 24 y $31^{\circ} \mathrm{C}$, rangos especificados por el fabricante para el adecuado funcionamiento de la unidad.

3) Condiciones de confort térmico del espacio según norma (ISO 7730, 2005), con PMV (Predicción del voto promedio) entre \pm 1 (-1 confortablemente caliente, 1 confortablemente frío).

4) Capacidad de la máquina de enfriamiento solar, suficiente para cubrir los requerimientos de demanda de enfriamiento total del conjunto de 5 viviendas. 


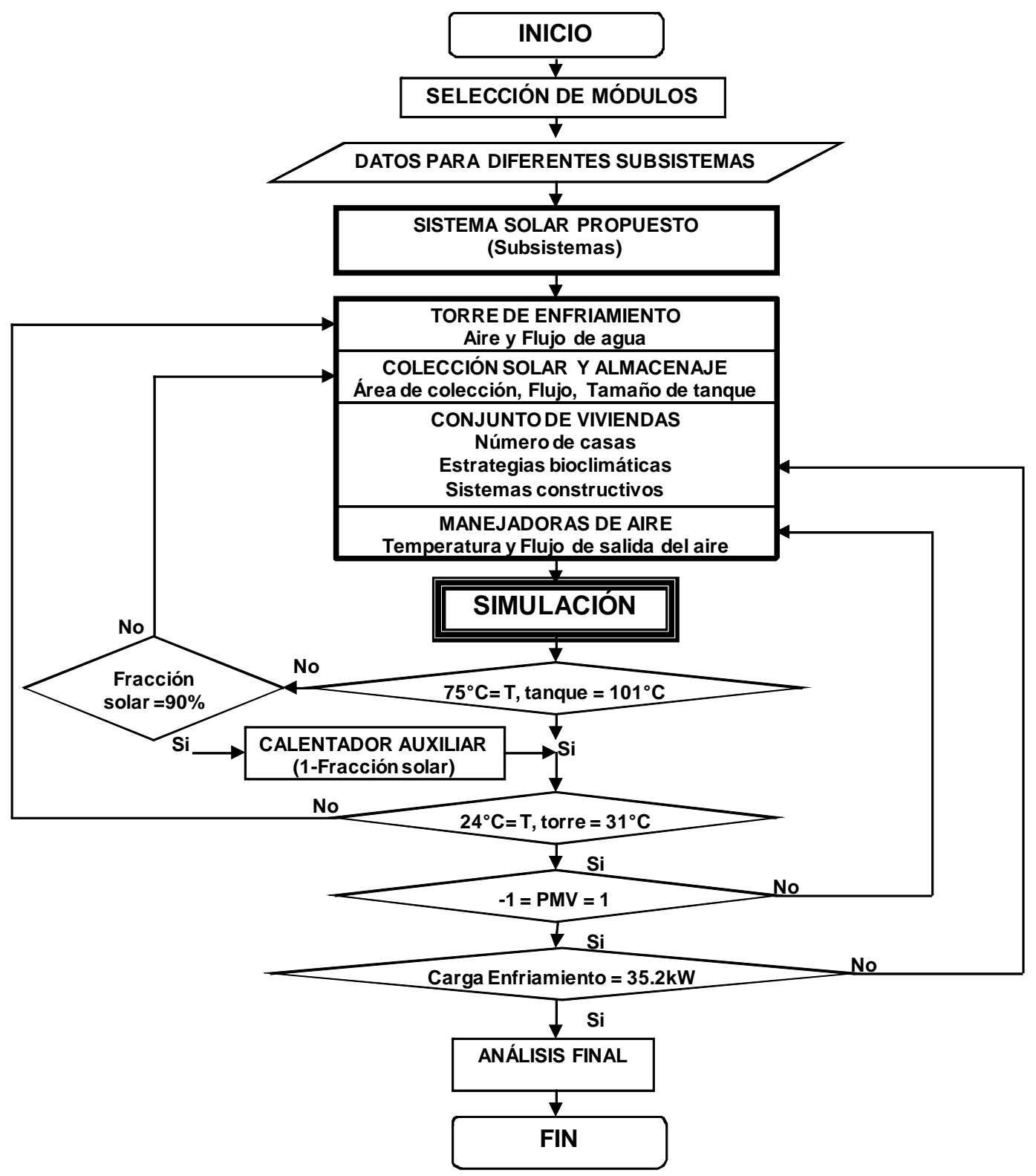

Fig. 2: Diagrama de flujo para dimensionamiento de sistema y simulación

\section{RESULTADOS Y DISCUSIÓN}

En ésta sección, se realiza el estudio de comportamiento operativo del SES durante el periodo de máxima demanda de enfriamiento. Para el análisis de resultados se describen las características constructivas de la envolvente de la edificación, las condiciones climáticas del lugar y la disponibilidad del recurso solar. Posteriormente se presenta el comportamiento de cada uno de los subsistemas y se finaliza con el análisis a detalle de los parámetros importantes del sistema global.

\section{Edificación}

Las condiciones de confort térmico de la edificación dependen del clima, propiedades térmicas de los sistemas constructivos y de las cargas internas. Las condiciones del aire del espacio, se evalúan a partir del componente 56, y se determina la temperatura y humedad relativa de las viviendas. En la tabla 2 se muestra el prototipo de viviendas simulado, con un área construida de $43.5 \mathrm{~m}^{2}$, cuenta con dos recámaras, espacio múltiple sala-comedor-cocina y un baño. La altura de piso a techo es de $2.40 \mathrm{~m}$ y un volumen de construcción de $106 \mathrm{~m}^{3}$. El área de cada una de las superficies de la envolvente arquitectónica y las capas que componen los sistemas constructivos se muestran en la tabla 2. 
Tabla 2: Prototipo, sistemas constructivos y áreas de superficies de la envolvente del edificio

\begin{tabular}{|c|c|c|c|}
\hline Elemento & Área $\left(\mathrm{m}^{2}\right)$ & Sistemas constructivos & Prototipo \\
\hline Piso & 43.5 & \multirow{12}{*}{$\begin{array}{l}\text { Muros: Bloque concreto } 0.12 \mathrm{~m} \text {, con } \\
0.01 \mathrm{~m} \text { mortero al norte, sur y este. Oeste } \\
\text { igual, con } 0.02 \mathrm{~m} \text { de poliestireno. } \\
\text { Techos: Concreto y casetón de } \\
\text { poliestireno } 0.15 \mathrm{~m} \text { mas } 0.01 \mathrm{~m} \text { de } \\
\text { mortero, } 0.05 \mathrm{~m} \text { de poliestireno en } \\
\text { exterior, membrana y lámina asfáltica. } \\
\text { Piso: Concreto armado de } 0.10 \mathrm{~m} \text {, con } \\
\text { loseta cerámica, mortero cemento - } \\
\text { arena de } 0.01 \mathrm{~m} \text {. } \\
\text { Puertas: Madera de pino (tipo tambor), } \\
\text { de } 0.03 \mathrm{~m} \text { de espesor. } \\
\text { Ventanas: Vidrio claro sencillo de } 0.003 \\
\mathrm{~m}\end{array}$} & \\
\hline Techo & 43.5 & & \\
\hline Muros Norte & 13.81 & & \\
\hline Ventanas norte & 1.80 & & \\
\hline Puerta & 2.05 & & \\
\hline Muros Sur & 15.39 & & \\
\hline Ventanas sur & 2.00 & & \\
\hline Muros Este & 25.13 & & \\
\hline Muros oeste & 24.15 & & \\
\hline Ventanas oeste & 0.24 & & \\
\hline Puerta & 1.64 & & \\
\hline Muros Interiores & 30.86 & & \\
\hline
\end{tabular}

Clima

El clima, es un factor importante para la operación del sistema de acondicionamiento de aire termosolar. Para el análisis climático de Mexicali, Baja California, se consideró un archivo de año típico meteorológico generado por Gallegos et al. (2006) con datos medidos del 2000 al 2005, proporcionados por el Servicio Meteorológico Nacional.

La figura 3 muestra que la temperatura ambiente promedio diaria $\left(T_{\text {amb }}\right)$ es mayor a $30{ }^{\circ} \mathrm{C}$ del 16 de mayo al 30 de septiembre, sin embargo, el periodo crítico se da en los meses de julio y agosto con temperaturas promedio máximas de $38{ }^{\circ} \mathrm{C}$ y máxima extrema de $46{ }^{\circ} \mathrm{C}$. En lo que respecta a los registros de humedad relativa (HR), se observa que se cuenta con valores entre 60 y $80 \%$ con registros máximos durante el mes de septiembre. Por otro lado la disponibilidad de radiación solar $\left(R_{s \_ \text {HOR }}\right)$ en el periodo de verano es mayor en los meses de mayo y junio con una máxima radiación solar diaria integrada de $2,199 \mathrm{~kJ} / \mathrm{m}^{2}$

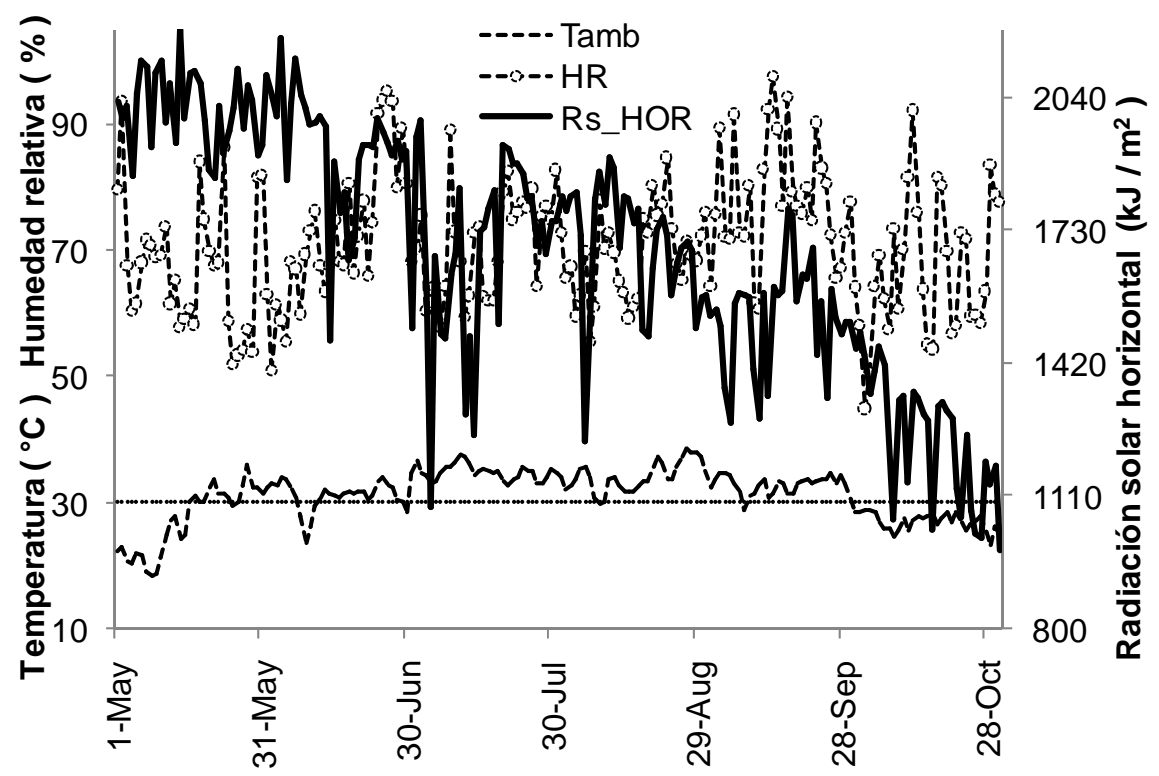

Fig. 3: Clima del periodo de requerimiento de enfriamiento

\section{Captación solar y almacenamiento térmico}

El sistema de colección solar está compuesto por $287 \mathrm{~m}^{2}$ de colectores de tubos evacuados, con una eficiencia de colección al origen de 0.87 . El área disponible en las viviendas es de $240 \mathrm{~m}^{2}$ por lo que es necesaria una pequeña extensión de techo para colocación de colectores. Con el área propuesta 
se aporta el 90\% de la energía demandada por el sistema de absorción, sin embargo, una mayor área no incrementa significativamente la fracción solar. En la figura 4 se puede observar que cuando se disminuye el área de colección se incrementa la demanda de calor auxiliar, por lo que se debe buscar un compromiso entre estos dos parámetros. Para obtener una operación continua durante todo el periodo de demanda de enfriamiento, se propone un tanque de almacenamiento de $24 \mathrm{~m}^{3}$, ya que con un tanque más pequeño la temperatura del líquido caloportador es menor a los requerimientos del sistema de absorción durante las horas nocturnas, lo cual coincide con los estudios realizados por Florides et al. (2002a). En la figura 5 se observa que durante julio y agosto, la demanda de energía del SES genera una disminución de la temperatura de entrada al arreglo de colectores $\left(\mathrm{T}_{\mathrm{e} \_\mathrm{col}}\right)$. Lo que provoca una mayor obtención de calor útil ( $\left.\mathrm{Q}_{\text {util }}\right)$. Sin embargo, es necesaria la aportación de calor auxiliar $\left(\mathrm{Q}_{\mathrm{aux}}\right)$ en los periodos críticos para la operación óptima del SES.

Por otro lado, en el periodo de mayo a junio la temperatura del tanque de almacenamiento térmico se incrementa por encima de los $100^{\circ} \mathrm{C}$; enviando el fluido caloportador a altas temperaturas al arreglo de colectores, debido a la baja demanda de energía por parte del SES. Esto hace que el agua enviada al sistema de colectores obtenga un bajo $\mathrm{Qutil}_{\text {en }}$ las horas con disponibilidad de radiación solar. El mismo efecto se repite en octubre, en el que se presenta el periodo con menor obtención de Qutil, ya que la $\mathrm{T}_{\mathrm{amb}}$ disminuye por debajo de los $30{ }^{\circ} \mathrm{C}$, requiriéndose menor enfriamiento en las edificaciones, lo cual se traduce en una menor demanda de energía por parte del SES, obteniéndose aún con una baja disponibilidad de radiación solar, altas temperaturas en el tanque de almacenamiento.

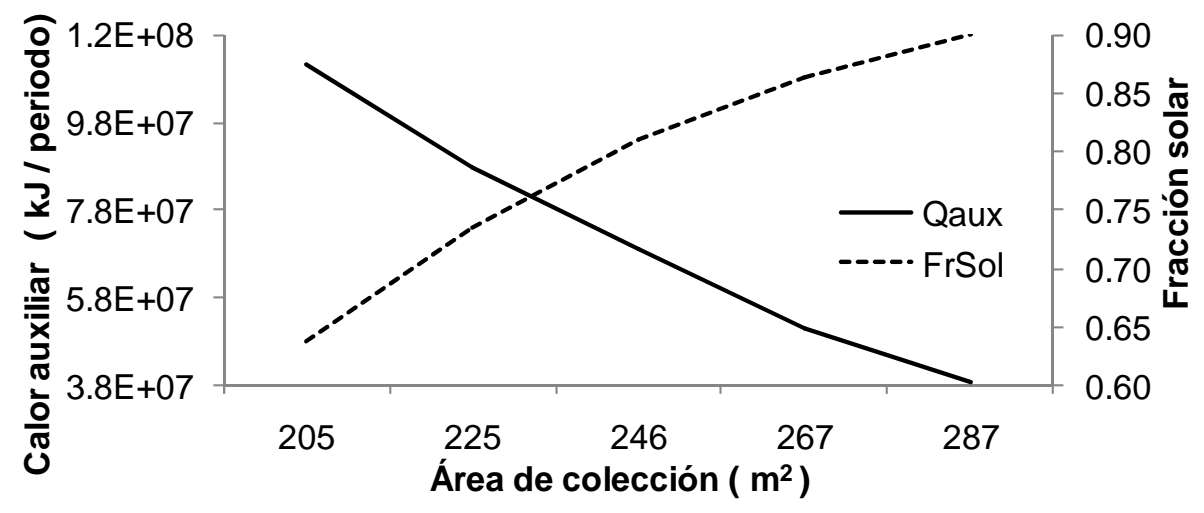

Fig. 4: Estudio de optimización de colección solar

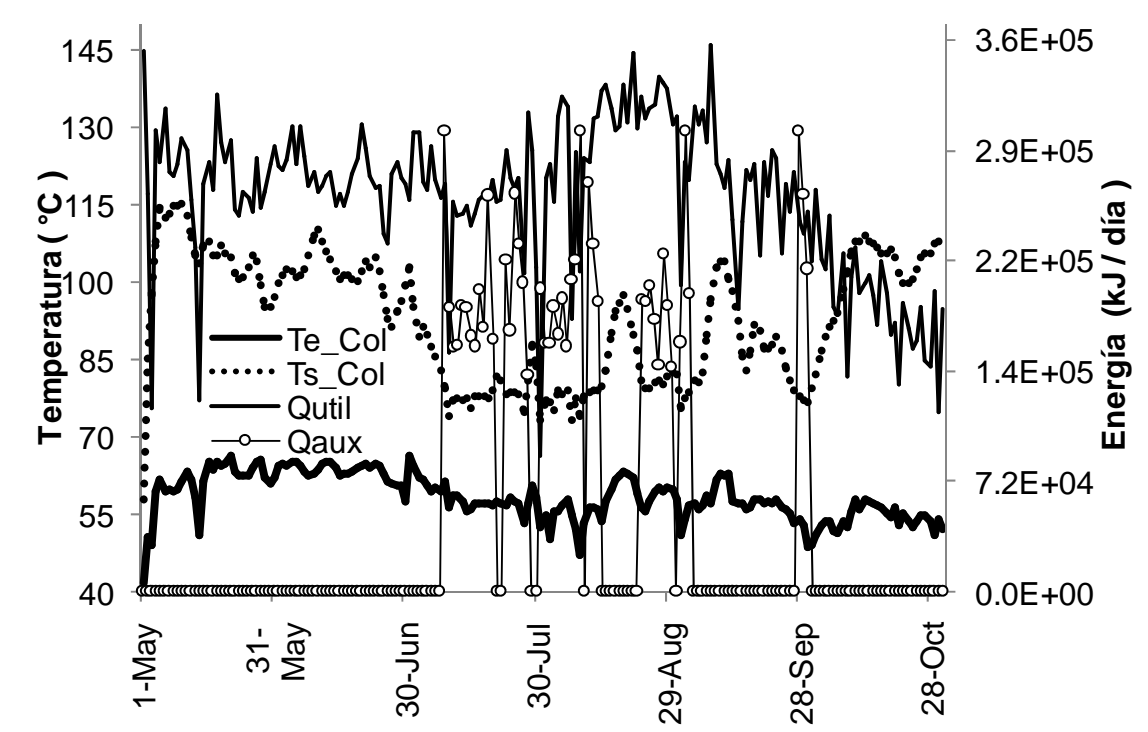

Fig. 5: Comportamiento del sistema de colección solar 


\section{Suministro de energía al sistema}

Para una correcta operación del SES, el fluido caloportador enviado del tanque de almacenamiento al generador ( $\left.T_{e \_G e n}\right)$, deberá estar entre 75 y $101^{\circ} \mathrm{C}$; de tal manera que se garantice un coeficiente de operación (COP) mayor a 0.5 y se produzca una adecuada ebullición de la solución diluida. Para tal efecto, se propone un sistema a gas natural, con una capacidad máxima de $180,720 \mathrm{~kJ} / \mathrm{hr}$.

Con la finalidad de obtener el mejor funcionamiento del SES a la máxima fracción solar ( $\left.F_{r_{-} s o l}\right)$, se realiza una serie de simulaciones exploratorias, fijando diferentes temperaturas de operación del calentador auxiliar. Los resultados se pueden observar en la figura 6 . El estudio muestra que la temperatura mínima óptima de entrada al generador es $80^{\circ} \mathrm{C}$, ya que es el punto en el que se obtiene la máxima facción solar (90\%) y como consecuencia, en la temporada solo se requiere de un $10 \%$ de $Q_{a u x}$ para cubrir la demanda de energía del SES.

Si se disminuye la temperatura de operación del calentador auxiliar al mínimo $\left(75^{\circ} \mathrm{C}\right)$, es necesario un $59 \%$ más de $\mathrm{Q}_{\text {aux }}$ respecto al requerido con operación óptima de $80{ }^{\circ} \mathrm{C}$. También se observa que al incrementar el punto de consigna a $100{ }^{\circ} \mathrm{C}$, se requiere del $4 \%$ más de Qux. Cabe señalar que cuando la temperatura de operación se maneja en $82^{\circ} \mathrm{C}$, no existe diferencia significativa en la $\mathrm{F}_{\mathrm{r}_{-} \text {sol, }}$, ni en la demanda de $Q_{a u x}$.

En la figura 7, se puede observar, que de julio a septiembre la $T_{e} \_$Gen se encuentra la mayor parte del tiempo entre 80 y $85^{\circ} \mathrm{C}$, algunos periodos con temperaturas superiores a los $90{ }^{\circ} \mathrm{C}$. Lo anterior es debido a que el decremento de $\mathrm{T}_{\text {amb }}$ provoca menor carga de enfriamiento en el conjunto de viviendas y hace que incremente la temperatura del fluido en el tanque de almacenamiento.

El mismo fenómeno se produce en mayo, junio y octubre, pero en esos casos la $T_{e}$ Gen excede el límite superior. Este excedente de energía, puede ser utilizado por un sistema de calentamiento de agua para uso domestico, además de utilizarse para calefacción de las viviendas a través de piso radiante; obteniendo de esta forma, un sistema integral de agua caliente, calefacción y enfriamiento durante el periodo anual, con esta integración se hace más rentable el sistema de colección y almacenamiento solar.

El sistema de colección solar, tiene la mayor demanda de energía térmica ( $\left.Q_{r} A C\right)$ por parte del SES, en los meses de julio y agosto, lo que implica una extracción promedio de $1.5 \times 10^{5} \mathrm{~kJ} / \mathrm{día}$. Sin embargo, durante los meses de mayo, junio y septiembre disminuye a $1.3 \times 10^{5} \mathrm{~kJ} / \mathrm{h}$, debido a la reducción de $\mathrm{T}_{\mathrm{amb}}$; con valores promedio diarios ligeramente superiores a los $30^{\circ} \mathrm{C}$. Por otro lado, la demanda de enfriamiento en octubre, disminuye a $1.2 \times 10^{5} \mathrm{~kJ} /$ día, ya que la $\mathrm{T}_{\mathrm{amb}}$ promedio diaria es menor a $30^{\circ} \mathrm{C}$.

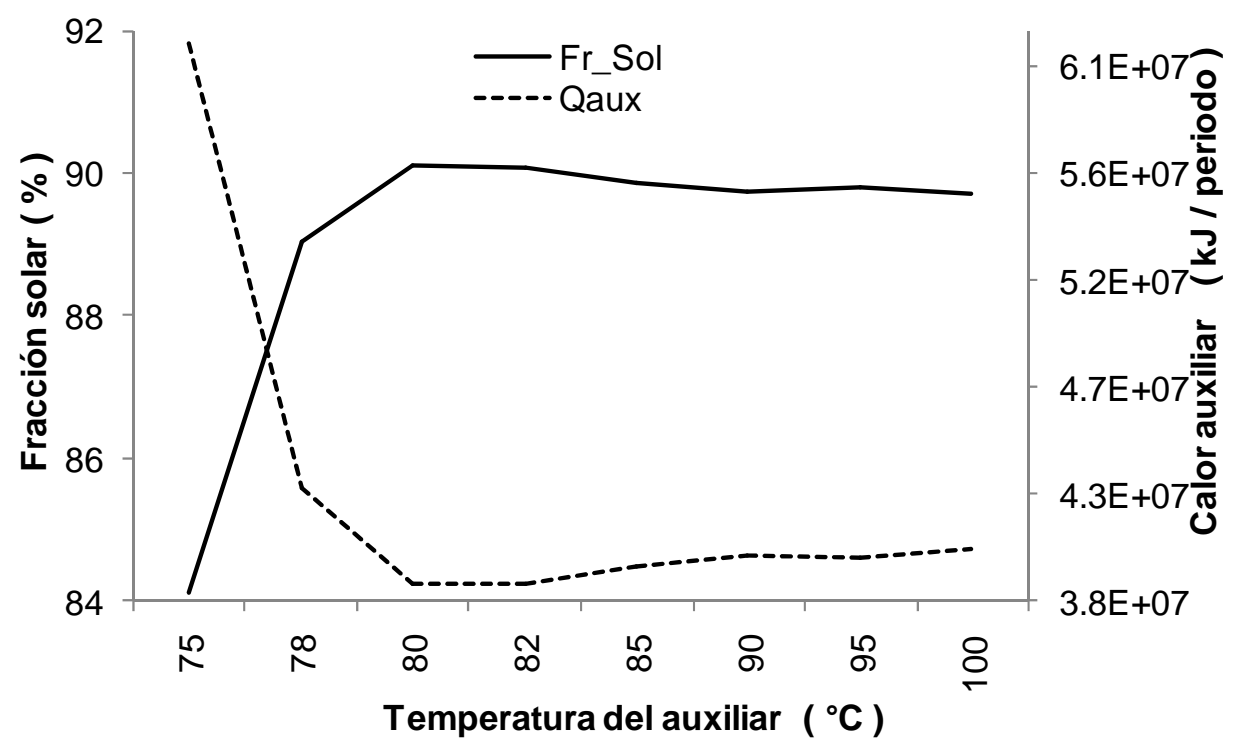

Fig. 6: Estudio de optimización del calentador auxiliar 


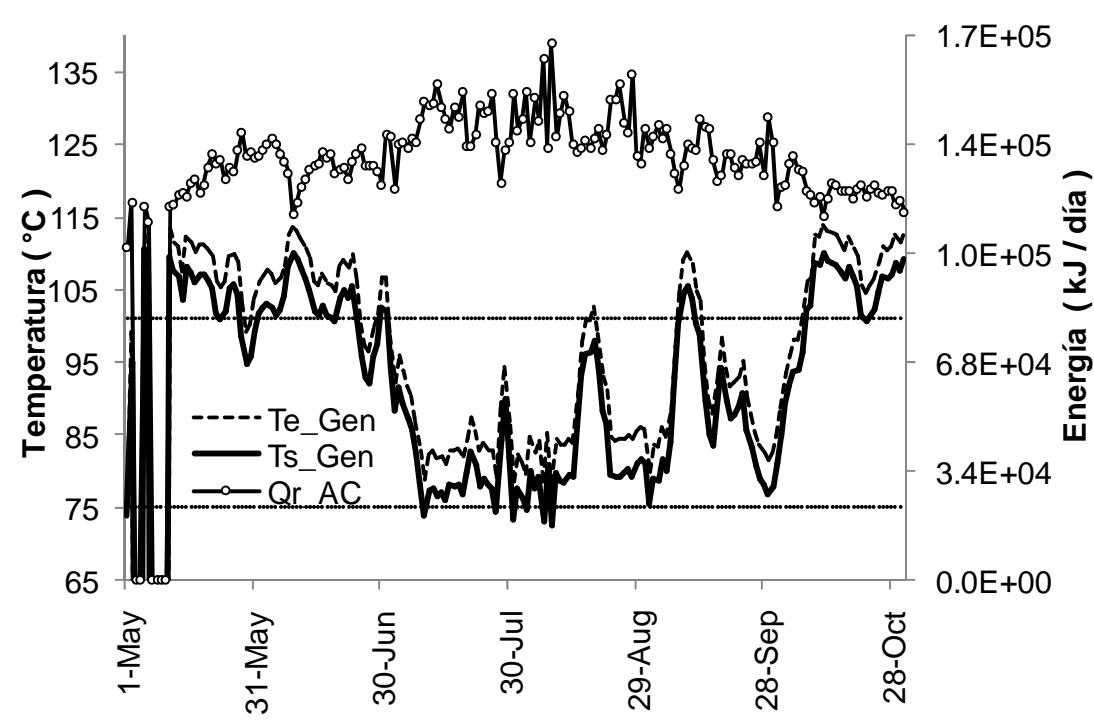

Fig. 7: Energía removida por el sistema de absorción

\section{Agua de enfriamiento}

En los sistemas de enfriamiento por absorción de $\mathrm{LiBr}-\mathrm{H}_{2} \mathrm{O}$, es importante un adecuado dimensionamiento de la torre de enfriamiento (TE), ya que el SES demanda temperatura de salida de la torre de enfriamiento $\left(T_{\mathrm{S}}\right.$ Trre $)$ entre 24 y $31{ }^{\circ} \mathrm{C}$, para garantizar el correcto funcionamiento de la unidad; además de evitar problemas de cristalización del LiBr. Para tal efecto se considera una TE de ciclo abierto, con un flujo de agua de $18,360 \mathrm{~kg} / \mathrm{h}$, para remover un máximo de energía $\left(\mathrm{Q}_{r_{-}}\right.$Trre $)$de $3.1 \times 10^{5} \mathrm{~kJ} / \mathrm{h}$. El flujo de aire es variable, desde un máximo de $7000 \mathrm{~m}^{3} / \mathrm{h}$ a un mínimo de $1,500 \mathrm{~m}^{3} / \mathrm{h}$.

Como se esperaba, el máximo $\mathrm{Q}_{\mathrm{r} \text { Trre }}$ se da en los meses de julio y agosto, con una extracción entre $2.4 \times 10^{5}$ y $2.7 \times 10^{5} \mathrm{~kJ} /$ día, de agua con temperatura promedio diaria de entrada $\left(\mathrm{T}_{\mathrm{e}}\right.$ Trre $)$ entre 31 y $33^{\circ} \mathrm{C}$, entregando al sistema una $\mathrm{T}_{\mathrm{s}_{-} \text {Trre }}$ promedio diaria entre 28 y $30^{\circ} \mathrm{C}$, como se puede observar en la figura 8.

El estudio de Chan et al. (2004), muestra que cuando $T_{\mathrm{s} \text { Trre }}$ entra a valores cercanos al límite de operación del sistema, se ve afectada la producción de agua fría, ya que genera un incremento de la concentración de la solución y disminuye la transferencia de calor del absorbedor; con lo que se provoca una disminución del potencial de absorción de la solución. Mayo y octubre son los meses con menor $\mathrm{Q}_{\mathrm{r}_{-} T r r e}$ y no representan ningún problema de cristalización del $\mathrm{LiBr}$, ya que $\mathrm{T}_{\mathrm{s}_{-} \text {Trre }}$ se encuentra entre 27 y $28^{\circ} \mathrm{C}$.

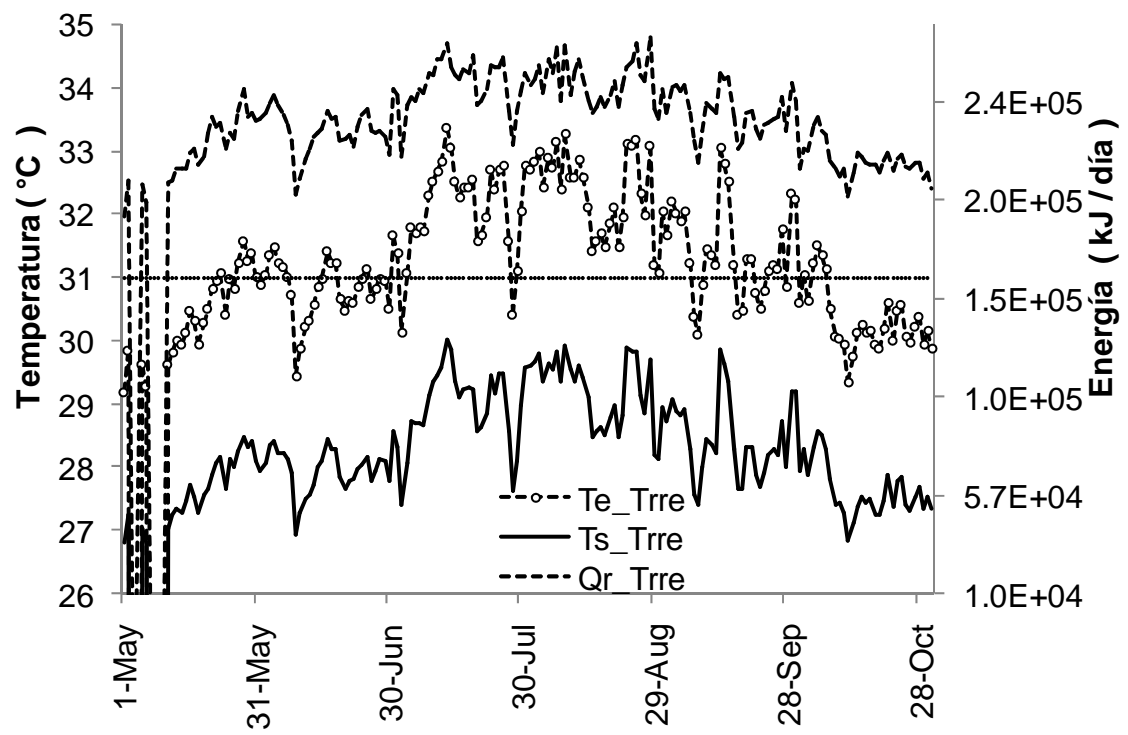

Fig. 8: Torre de enfriamiento 


\section{Agua fría}

La temperatura del agua fría que entra a las manejadoras de aire $\left(T_{\text {e_AF }}\right)$ se establece en $8{ }^{\circ} \mathrm{C}$, con temperatura de retorno $\left(\mathrm{T}_{\mathrm{S} \_\mathrm{AF}}\right)$ al sistema de absorción de $12.5^{\circ} \mathrm{C}$, después de retirar energía del aire $\left(Q_{r_{-}}\right.$ir $)$que proviene del conjunto de viviendas. En la figura 9, se muestra que en mayo, junio, septiembre y octubre la $\mathrm{T}_{\mathrm{e} \text { A }} \mathrm{f}$ opera en condiciones óptimas. Sin embargo, en los meses de julio y agosto la $T_{\text {e } A F}$ es mayor a la temperatura de diseño. Lo anterior debido a que el sistema de enfriamiento remueve del aire del espacio $1.0 \times 10^{5} \mathrm{~kJ} / \mathrm{h}$, cantidad de energía cercana a la capacidad nominal del SES $\left(1.3 \times 10^{5} \mathrm{~kJ} / \mathrm{h}\right)$. Además de que la $\mathrm{T}_{\mathrm{e}}$. Trre, cuenta con valores cercanos a los $31{ }^{\circ} \mathrm{C}$, límite superior de entrada. Esto trae como consecuencia, que la unidad de absorción trabaje en forma ineficiente y disminuya su capacidad.

\section{Aire frío}

Para climatización de los espacios de las viviendas, se utilizan manejadoras de aire que deshumidifican y enfrían el aire por medio de agua fría procedente del ciclo de absorción.

El subsistema de aire frío está compuesto por cinco unidades manejadoras, que retiran la energía del aire del espacio ( $Q_{\text {r_Air }}$ ) a través de 1,100 kg/h de agua fría, con un volumen de aire de 2,072 kg/h, a una capacidad nominal de enfriamiento de $31,652 \mathrm{~kJ} / \mathrm{h}$. La climatización del espacio se realiza por medio de aire frío con temperatura de $16{ }^{\circ} \mathrm{C}\left(\mathrm{T}_{\mathrm{s}_{-} \text {AirMJ }}\right)$, para mantener la temperatura del espacio entre 23 y $28^{\circ} \mathrm{C}$.

En la figura 10, se puede apreciar que la $T_{s_{-} \text {Airmu }}$ suministrado a las viviendas se mantiene entre 16 y $17{ }^{\circ} \mathrm{C}$, con lo que se obtienen temperaturas del aire de las viviendas ( $T_{\text {esp }}$ ) a un promedio diario de entre 23.5 y $25^{\circ} \mathrm{C}$. Los periodos de mejor operación de las manejadoras de aire se dan del 1ro de mayo al 30 de junio y desde el 9 de septiembre al 31 de octubre, a partir de una $\mathrm{T}_{\mathrm{s} \_ \text {Airmu }}$ de $16{ }^{\circ} \mathrm{C}$, que remueve un máximo de $9.8 \times 10^{4} \mathrm{~kJ} / \mathrm{h}$.

Por otro lado, el periodo crítico en la operación del SES, se da del 1ro de julio al 8 de septiembre, con una $\mathrm{T}_{\mathrm{s} \_ \text {Airmu }}$ promedio diaria de $17^{\circ} \mathrm{C}$, que retira un promedio diario de $1.0 \times 10^{5} \mathrm{~kJ} / \mathrm{h}$. En lo que respecta a las temperaturas proporcionadas por la calidad del aire de las manejadoras, en la figura 11 , se puede observar que la $\mathrm{T}_{\text {esp }}$ se mantiene entre 24 y $25^{\circ} \mathrm{C}$. Además de que la $\mathrm{HR}$ de las viviendas se mantiene entre 45 y $65 \%$ la mayor parte del tiempo. La mayor HR del espacio se presenta en el mes de octubre con valor promedio diario máximo de $75 \%$.

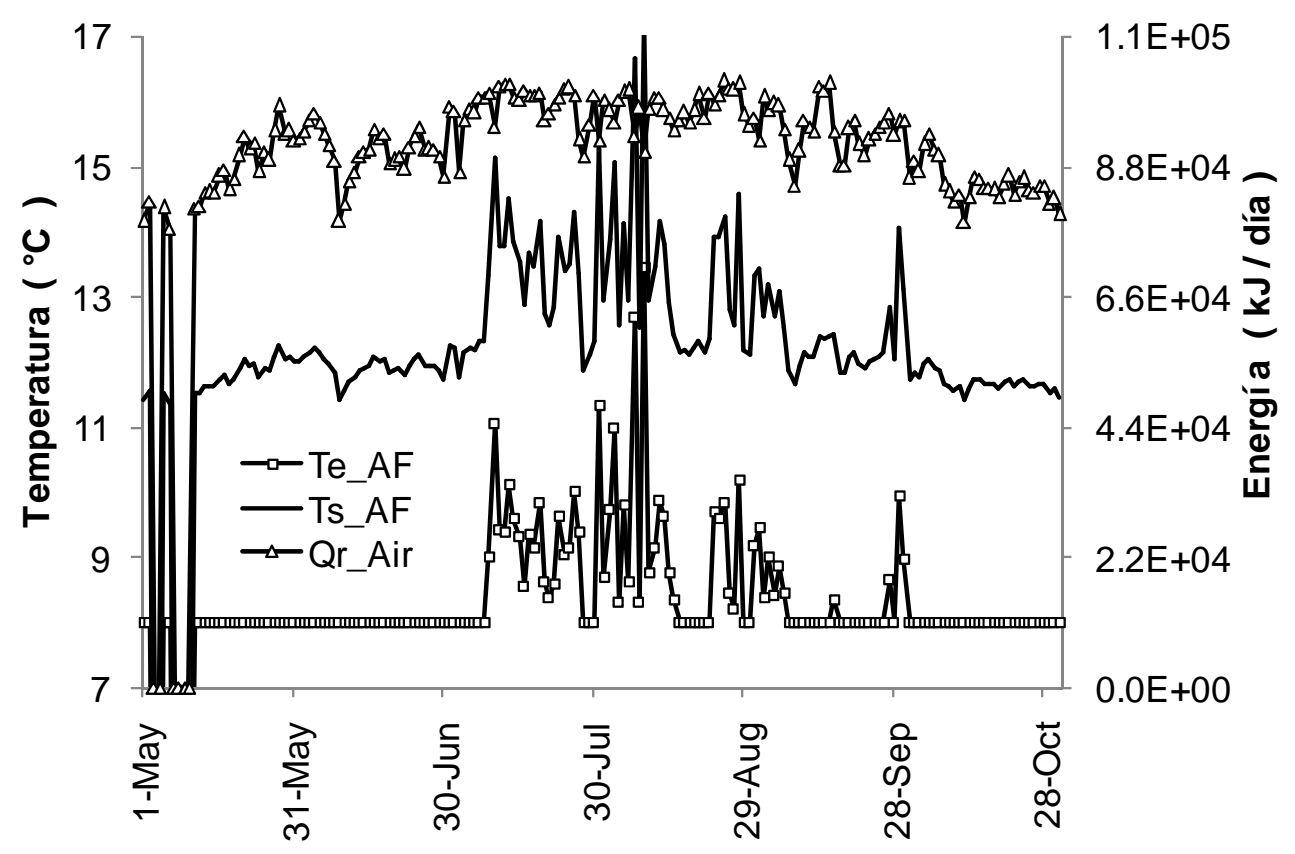

Fig. 9: Energía removida en agua fría 


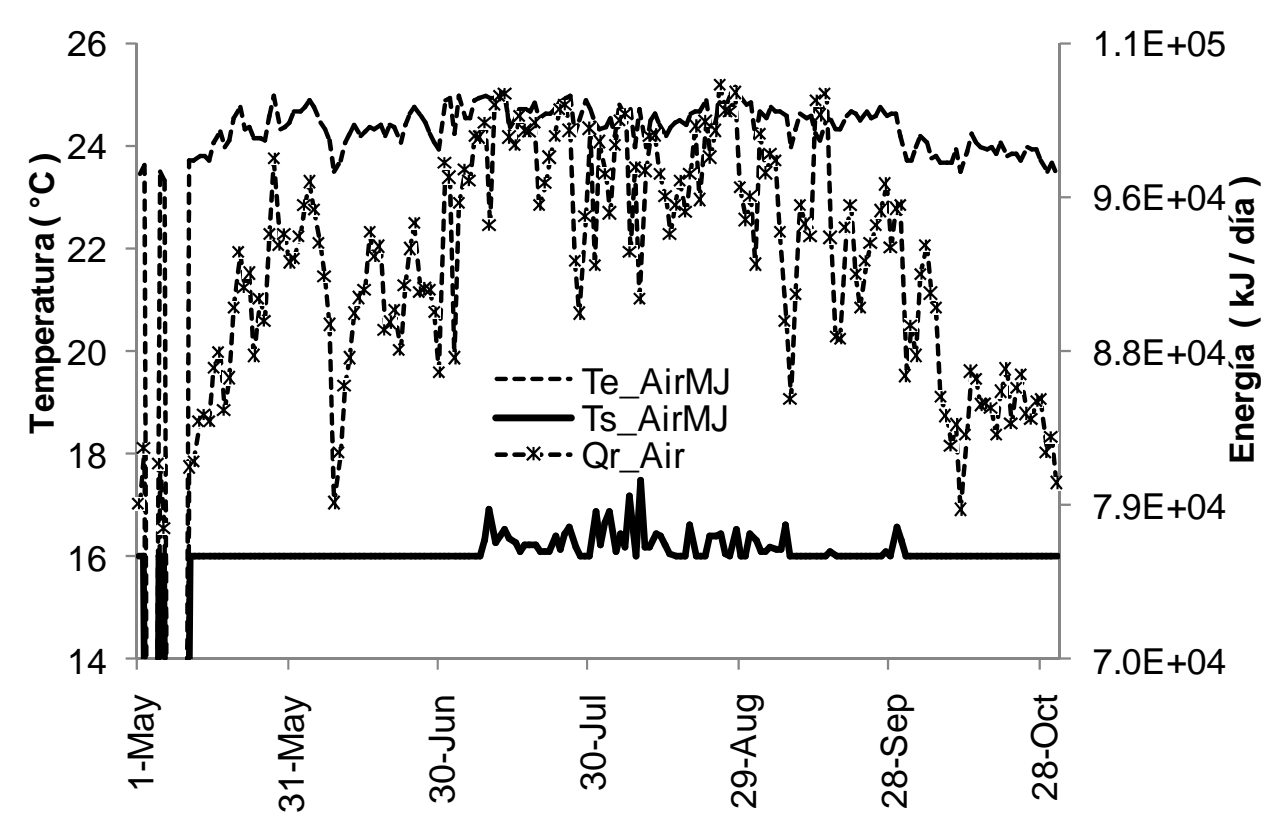

Fig. 10: Energía removida por el aire frío

En la figura 11, se observa que la predicción del voto promedio del espacio $\left(\mathrm{PMV}_{\text {esp }}\right)$ propiciado por la temperatura del aire de las unidades manejadoras está entre 0.2 y 0.4 , la mayor parte del periodo de máxima demanda de enfriamiento. Lo que indica que de acuerdo a la escala de confort térmico según ISO:7730 (2005) los espacios se encuentran en condiciones de confort óptimo según los limites siguientes: -3 Extremadamente frío, -2 Demasiado frío, -1 Confortablemente frío, 0 Confortable, 1 Confortablemente caliente, 2 Demasiado caliente, 3 Extremadamente caliente. Lo anterior considerando que los usuarios visten ropa ligera equivalente a 0.6 clo, con actividad metabólica ligera de 1.2 met.

Existen algunos periodos en los cuales el $\mathrm{PMV}_{\text {esp }}$ incrementa ligeramente a valores de entre $0.47 \mathrm{y}$ 0.45 , esto debido a que la unidad de enfriamiento no envía agua fría con suficiente gradiente de temperatura respecto a la temperatura del aire interior del espacio.

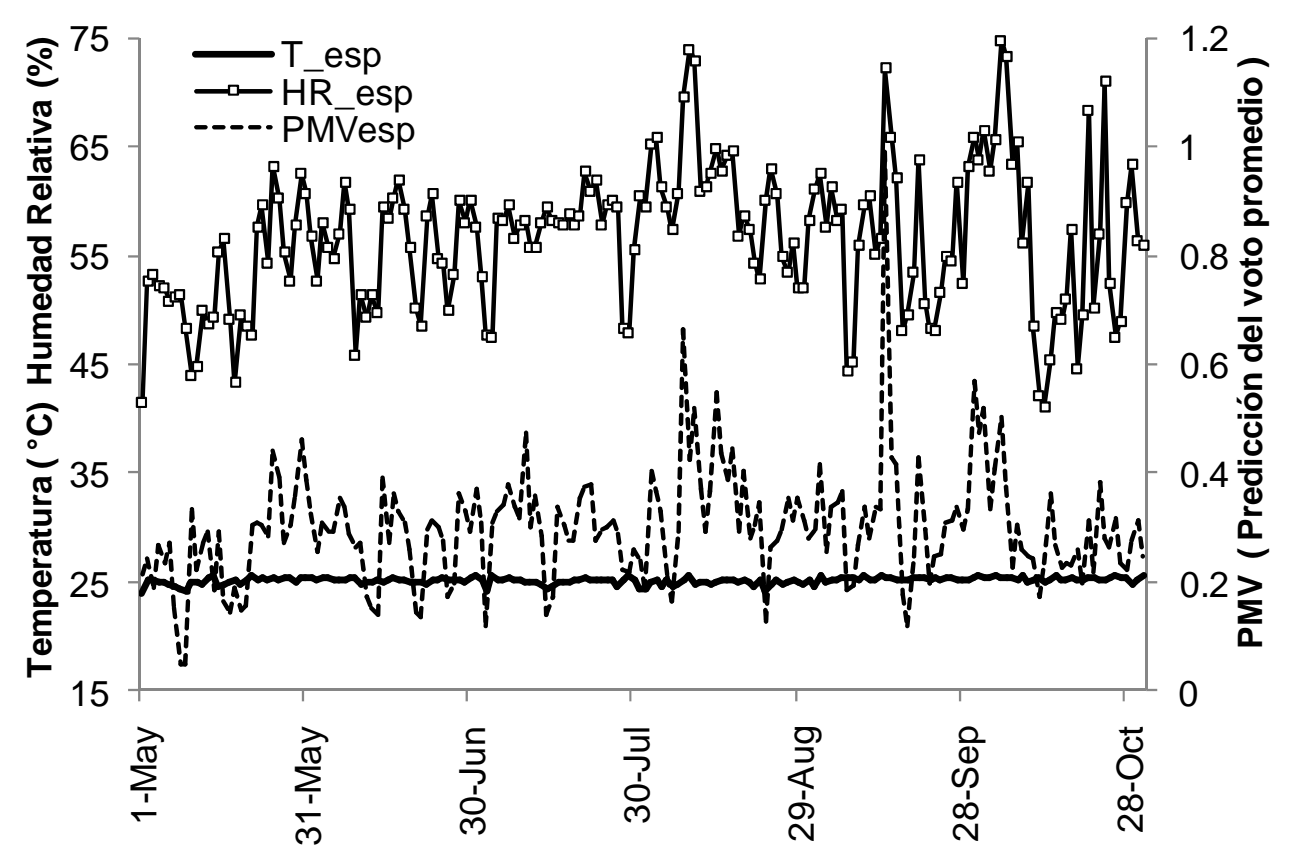

Fig. 11: Climatización del espacio de las viviendas 


\section{Eficiencia total del sistema}

A la combinación de la eficiencia del campo de colectores y del COP del ciclo de absorción se le conoce como eficiencia total del sistema o $\mathrm{COP}_{\text {solar }}$ y se obtiene a partir del producto de ambos (García-Casals, 2006). Entre los factores que afectan al COP solar están: temperatura ambiente, humedad relativa, área de captación solar, ángulo de incidencia con respecto al campo de colectores, entre otros.

Cuando se equilibra la eficiencia del sistema de colectores $\left(\mathrm{E}_{\mathrm{ff} \_ \text {col }}\right)$ con el COP del ciclo de absorción, se adquiere el máximo $\mathrm{COP}_{\text {solar }}$. Esto significa un mayor aprovechamiento de la energía solar para producción de agua fría en el SES.

En la figura 12, se puede observar que el $\mathrm{COP}_{\text {solar }}$ promedio diario del sistema simulado durante los meses críticos de julio y agosto, se encuentra entre 0.28 y 0.38 , esto debido al clima y la alta demanda de enfriamiento, que disminuye la temperatura del tanque de almacenamiento térmico al mínimo permitido para operación de la unidad de absorción. Lo anterior genera una buena obtención de $\mathrm{Q}_{\text {util }}$ del campo de colectores, e incrementa la eficiencia de captación solar.

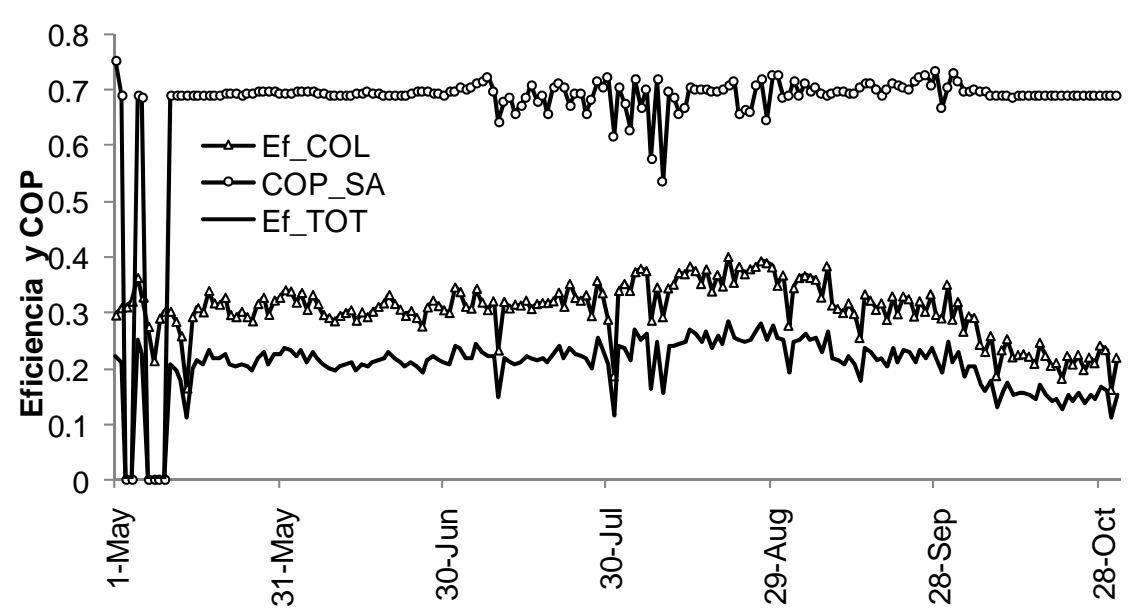

Fig. 12: Comportamiento total del sistema

\section{CONCLUSIONES}

Los resultados muestran que el sistema propuesto es técnicamente factible, ya que cubre la demanda de enfriamiento durante el periodo de verano, a través de un área de colección solar similar a la disponible en el conjunto de viviendas.

Se demuestra que la unidad de absorción trabaja con eficiencias (COP) en el rango especificado por el fabricante, de 0.5 a 0.8 , a partir del $90 \%$ de energía proporcionada por el campo de colectores solares y cumple con las restricciones requeridas.

El uso de estos sistemas de enfriamiento repercute en una disminución de contaminación ambiental ya que solamente es necesaria la quema de combustibles fósiles (gas natural) en un 10\%. Por lo tanto el uso de estos sistemas debería ser ampliamente utilizado para climatización de espacios en lugares con alta disponibilidad de recurso solar.

El dimensionamiento propuesto de la TE, permite operar en condiciones óptimas al sistema, ya que proporciona agua de enfriamiento de entre 24 y $31^{\circ} \mathrm{C}$, rango especificado por el fabricante de la unidad de absorción.

Los resultados de la simulación muestran que el aire frío suministrado a las viviendas, propicia condiciones de confort térmico, según la escala ISO 7730, para usuarios con una actividad metabólica de 1.2 met y factor de arropamiento de 0.6 clo. 


\section{AGRADECIMIENTOS}

Al Consejo Nacional de Ciencia y Tecnología (CONACYT) por la beca de doctorado; y a la Universidad Autónoma de Baja California (UABC), a través del Instituto de Ingeniería y la Facultad de Arquitectura y Diseño, por el apoyo académico.

\section{REFERENCIAS}

Assilzade, F., S. Kalogiroub, Y. Alia y K. Sopiana; Simulation and optimization of a $\mathrm{LiBr}$ solar absorption cooling system with evacuated tube collector. Renewable Energy: 30, 1143-1159 (2005).

Chan, W., J. Jin y T. Yong; Energy consumption characteristics of an absorption chiller during the partial load operation. International Journal of Refrigeration: 27, 948-954 (2004).

Florides, G., S. Kalogirou, S. Tassou y L. Wrobel; Modelling, simulation and warming impact assessment of a domestic-size absorption solar cooling system. Applied Thermal Engineering: 22, 1313-1325 (2002a).

Florides, G., S. Kalogirou, S. Tassou y L. Wrobel; Modelling and simulation of an absorption solar cooling system for Cyprus. Solar Energy: 72, 43-51 (2002b).

Gallegos, R., A. Luna, N. Velázquez y G. Bojórquez; Simulation study on the limitations of the use of evaporative cooling for air conditioning in hot arid climate of Mexicali. Mexican National Solar Energy Week Conference. Proceedings of ANES/ASME Solar Joint 2006,1-4, Veracruz, México. October 2-6 (2006)

García-Casals, X; Solar absorption cooling in Spain: Perspectives and outcomes from the simulation of recent installations. Renewable Energy. 31, 1371-1389 (2006).

Hans-Martin, H; Solar assisted air conditioning of buildings-an overview. Applied Thermal Engineering: 27, 1734-1749, (2007)

ISO:7730; Ergonomics of the thermal environment-Analytical determination and interpretation of thermal comfort using calculation of the PMV and PPD indices and local thermal comfort criteria, 152, Switzerland (2005).

Papadopoulos, A., S. Oxizidis y N. Kriakis; Perspectives of solar cooling in view of the developments in the air-conditioning sector. Renewable and Sustainable Energy Reviews: 7, 419-438, (2003).

Srikhirin, P., S. Aphornratana y S. Chungpaibulpatana; A review of absorption refrigeration technologies. Renewable and Sustainable Energy Reviews: 5, 343-372 (2001).

Syed, A. y otros seis autores; A novel experimental investigation of solar cooling system in Madrid. International Journal of refrigeration: 28, 859-871 (2005). 